\title{
Modeling and Generating Tone Contour with Phrase Intonation for Mandarin Chinese Speech
}

\author{
Zhizheng Wu* ${ }^{1,2}$, Yao Qian ${ }^{1}$, Frank K. Soong ${ }^{1}, \quad$ Bo Zhang ${ }^{2}$ \\ ${ }^{1}$ Microsoft Research Asia, Beijing $\quad{ }^{2}$ College of Software, Nankai University \\ wuzhizheng@gmail.com, \{yaoqian, frankkps\}@microsoft.com, \\ zhangb@tedmail.nankai.edu.cn
}

\begin{abstract}
This paper models Fo curves with discrete cosine transform (DCT) representations on both syllable-level tone and phrase-level intonation for Chinese Mandarin speech. Decision trees growing with maximum likelihood (ML) and stopping with minimum description length (MDL) are used to cluster very rich context-dependent DCT models into generalized ones to predict unseen contexts in test robustly. Additionally, we propose to generate Mandarin tone contours by jointly optimizing Fo contours of syllable and phrase in ML sense. Experimental results on speaker-dependent continuous and speakerindependent isolated speech corpora show that the proposed approach can be able to generate Fo contour with high correlation coefficients of 0.92 and 0.82 respectively, measured between the original and generated $\mathrm{F} 0$.
\end{abstract}

Keywords-F0 modeling, F0 generating, DCT, Mandarin tone, Intonation

\section{INTRODUCTION}

Chinese Mandarin as well as other Chinese dialects is known as a monosyllabically paced tonal language. Tone is indispensable for lexical access and disambiguation of homonyms in Mandarin. Fundamental frequency (F0) is the most important acoustic correlate of tone in spoken Mandarin. There have been numerous studies on F0 modeling [1-8]. These studies are roughly around three issues.

F0, a continuous variable, only exists in the voiced region of speech signals. In unvoiced segments, no F0 is observed. The discontinuity of F0 between voiced and unvoiced transition is the first issue to be considered. Heuristic approaches like interpolating F0 in unvoiced segments were proposed to get around discontinuity problem [6,7]. However, the artificially interpolated F0s do not reflect the actual tone and the voicing/unvoicing information, which is critical to F0 trajectory generation, is lost in these heuristic approaches. Multi-Space Distribution (MSD) [9], which models discrete and continuous probability spaces, has been proposed to model the partially continuous F0 contours in a statistically compact and rigorous manner. On the other hand, only voiced segments taken from a corpus with the transcription of phone, syllable and word boundaries by performing a forced alignment are used for training the F0 model [4].

Parametric representation is another issue. F0 movements directly represent the patterns of tone contours. Original F0s or several target F0 points from original curves can be used for tone contour modeling [1-4]. F0 is a highly variable acoustic feature from different speakers or even same speaker from time to time. Effective normalization, e.g. taking logarithm or removing sentence-level mean, is generally used to minimize the undesirable F0 fluctuations. However, non-parametric model is not so inherent to predict smoothed F0 trajectories. The post-processing or generation with dynamic feature constraint $[1,4,15]$ is used to compensate for it. Parametric models usually transform the original F0s into some parametric representations like exponential decay function, natural cubic spline and discrete cosine transform [5-8]. After modeling, the parameters are first predicted, then the tone contours are generated from these parameters. Parametric models can not only predict smoothed tone contour directly but also approximate the long term contour like intonation precisely.

The additive structure of F0 is commonly observed across different languages. The theory of superposition of lexical tone and phrase or sentence intonation or the so-called "small ripples on top of big waves" was first proposed by Chao [10] and empirically confirmed in Mandarin. F0 modeling and generating with the hierarchical superposition of different intonation components is the third issue. Multi-layer models have been generally used to model F0s [3-7]. Actually, the superposition of F0 contours predicted from different models and presented by different lengths is not so trivial.

In this paper, we first investigate the parametric representations for F0 contours, then use discrete cosine transform (DCT) to parameterize the F0 curves on both syllable and phrase levels. After tying rich context-dependent DCT coefficients by decision with maximum likelihood (ML) criterion, we propose to generate tone contour by jointly optimizing F0 contours of syllable and phrase in ML sense.

\section{PARAMETRIC REPRESENTATION}

Two conventional parametric forms, natural cubic spline and discrete cosine transform, are investigated for the parametric representation of F0 curves. A natural cubic spline (NCS) [11] is a piece-wise cubic function defined in terms of the points called knots $x_{1}, \ldots, x_{k}$, satisfying $x_{1}<x_{2}<\cdots<$ $x_{k-1}<x_{k}$ and the first and second derivatives are continuous at each intermediate knot $x_{2}, \ldots, x_{k-1}$. The so-called a "natural" cubic spline has additional constraints namely that the second derivatives of the first and last knots are zeros. The discrete cosine transform (DCT) is a linear, invertible function. It

\footnotetext{
*Work performed as an intern in the Speech Group, Microsoft Research
} Asia 
expresses a finite signal in terms of a sum of cosine functions oscillating at different frequencies. The most common DCT definition is

$$
c_{n}=\frac{2}{\mathrm{M}} \sum_{m=0}^{M-1} s_{m} \cos \left[\frac{\pi}{\mathrm{M}} n\left(m+\frac{1}{2}\right)\right], n=0, \ldots, N-1
$$

where $s_{0}, \ldots, s_{M-1}$ is a finite signal of length $\mathrm{M}$ and represented by $\mathrm{N}$ coefficients of DCT $, c_{0}, \ldots, c_{N-1}$. Similarly, the inverse transformation is defined as

$$
s_{m}=\frac{1}{2} c_{0}+\sum_{n=1}^{N-1} c_{n} \cos \left[\frac{\pi}{\mathrm{M}} n\left(m+\frac{1}{2}\right)\right], \quad m=0, \ldots, M-1
$$

F0 curves extracted from 18,000 Mandarin syllables in a female continuous speech corpus are used to check the fitting performances of NCS and DCT. Figure 1 shows the fitting errors in terms of root mean square error (RMSE) with increasing the number of DCT coefficients and NCS knots. DCT outperforms the NCS in the lower coefficients/knots. As the number of DCT coefficients and NCS knots used increases, the contours represented by DCT and NCS both rapidly approach the original F0 contours.

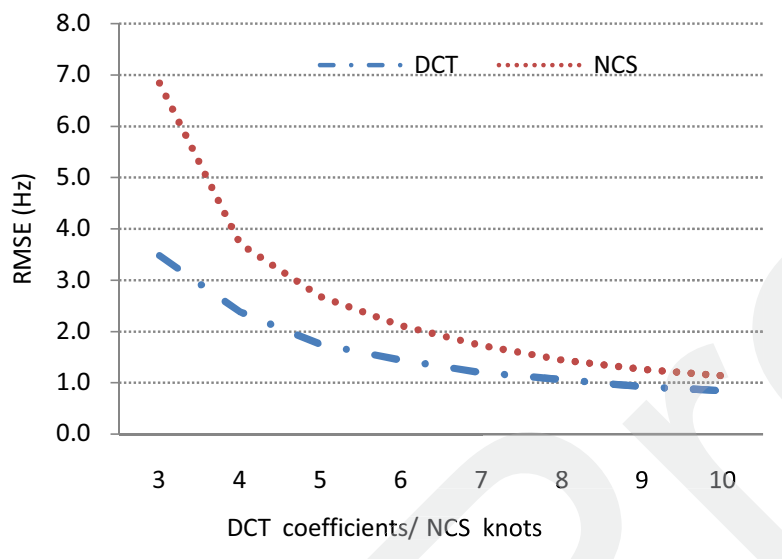

Figure 1. The fitting errors of Mandarin F0 contours by NCS and DCT

DCT is a sum of smooth functions with orthogonal transform coefficients which can be modeled independently. The first DCT coefficient is the mean of input signal and the rest coefficients are the weights for cosine functions with different wavelengths. They can represent the mean and the shape of F0 contours on different intonation components. In addition, the orthogonality property of DCT basis function can reduce computation complexity. Therefore, we use DCT for the parametric representation of $\mathrm{F} 0$ contours.

\section{PREPARE Your PAPER BEFORE STYLING}

F0 contours on syllable and phrase levels, which corresponds syllable tone and phrase intonation, are represented by DCT parameters. Classification and regression trees (CART), an effective data mining tools which can efficiently handle messy data, missing values, or predictor variables measured in different scales, is used to clustering those parameters. We use ML as a splitting criterion and minimum description length (MDL) [12] as a stopping criterion in decision tree growing. To capture tone co-articulation effects and generate the smoothed tone contours across syllables, the dynamic features of first coefficient of DCT, $c_{0}$, which represent the temporal information of syllables, are used for tone contour modeling.

In the generation part, tone contour is generated with dynamic feature constraint in the ML sense. For a given DCT model $\lambda$, it determines a sequence of tone contours, $S=$ $S_{0}^{M-1}, \ldots, S_{T-1}^{M-1}$, which maximizes $\log P(0 \mid \lambda)$ with respect to $O=W D S$, i.e.,

$$
\frac{\partial \log P(W D S \mid \lambda)}{\partial S}=0
$$

we obtain

$$
(W D)^{T} U^{-1} W D S=(W D)^{T} U^{-1} M
$$

where $s_{i}^{M-1}$ is F0 vector of syllable i; $D$ is DCT transform matrix; $U$ and $M$ are covariance matrix and mean vector of DCT coefficients; $W$ is delta and delta-delta coefficient matrix of first DCT coefficient.

As we know F0 has hierarchical superposition structure. Tone contours can be estimated more precisely if they can be regulated by the F0 information of longer intonation components like phrase. The likelihood of the tone contours is jointly maximized in conjunction with weighted likelihoods of phrase contours. It maximizes

$$
\log P\left(O_{p} \mid \lambda_{p}\right)^{\alpha} * P\left(O_{s} \mid \lambda_{s}\right)
$$

with respect to $O_{p}=D_{p} A S$ and $O_{s}=W D_{s} S$. Similar to Equation 3, we obtain the solution as

$$
\begin{aligned}
& \left\{\alpha\left[\left(D_{p} A\right)^{T} U_{p}^{-1} D_{p} A\right]+\left(W D_{s}\right)^{T} U_{s}^{-1} W D_{s}\right\} S \\
& =\alpha\left[\left(D_{p} A\right)^{T} U_{p}^{-1} M_{P}\right]+\left(D_{s} W\right)^{T} U_{s}^{-1} M_{s}
\end{aligned}
$$

where $D_{p}$ and $D_{s}$ are the DCT matrices for F0 contours on phrase and syllable levels; $A$ is the matrix to get the mean of F0s on each syllable; $U_{p}, U_{s}, M_{P}, M_{s}$ are covariance matrices and mean vectors of DCT coefficients on phrase and syllable levels. $\alpha$ is the parameter to weight the likelihood of phraselevel DCT model. When $\alpha=0$, only syllable-level DCT model is considered.

\section{EXPERIMETNS AND RESULTS}

\section{A. Experimental setup}

Two Mandarin corpora are used in our experiments. One is speaker-dependent continuous speech corpus which was recorded by a professional female in broadcast news style. It consists of 2487 training, 299 developing and 298 testing sentences. Another one is speaker-independent isolated speech corpus. The training set contains 1767 syllables from 5 males and 5 females. The developing and testing sets are both composed of 100 syllables. Speech signals in both corpora are 
sampled at $16 \mathrm{kHz}$, windowed by a $25-\mathrm{ms}$ window with a $5-\mathrm{ms}$ shift. F0 is extracted on a short-time basis by applying the robust algorithm for pitch tracking (RAPT) [13] and normalized by the mean of sentence F0 contours.

Considering the length of voiced part in some syllables can be less than $50 \mathrm{~ms}$ and the fitting errors is slowly reduced after 7 coefficients shown in Figure 1, we use 7 DCT coefficients, delta and delta-delta features of first DCT coefficients of proceeding, current and following syllables for representing syllable-level F0 contour. Tone-dependent decision trees are built for clustering those DCT coefficients. The corresponding question set for decision tree growing includes tones and breaks on contextual quin-syllable; the position of syllable and word in phrase and sentence; the length of word and phrase; the continuity of F0 contours on contextual tri-syllable. On phraselevel, 3 DCT coefficients are use to represent a contour that passes through the F0 mean of each constituent syllable. The questions used to build decision trees consist of the number of syllables in current phrase; position of the current phrase in the utterance; and number of phrase in the utterance.

\section{B. Experimental results and analysis}

To evaluate the performance of DCT models, we use them to generate F0 contours on syllable and phrase levels for testing data of speaker-dependent continuous and speaker-independent isolated speech corpora. The performance is measured objectively in terms of F0 distortions between the original and generated F0. The syllable boundary and duration are obtained by forced alignment with the acoustic models trained in our HMM-based speech synthesis system [14]. The voiced and unvoiced decisions are also given by our HMM-based TTS system. We measure the RMSE and correlation coefficient between original and generated F0 trajectories by the proposed approach. The evaluation results are given in the following subsections, respectively.

\section{1) Speaker-dependent continuous speech corpus:}

The tone contours are first generated by solving Equation 4 . Table I shows the RMSE and correlation coefficients between original and generated F0 contours with static and dynamic features. Although the dynamic features are only taken for the first DCT coefficient, it can improve the RMSE from $20.59 \mathrm{~Hz}$ to $19.54 \mathrm{~Hz}$. The tone contours are then generated by solving Equation 6, in which the likelihoods of the tone contours are jointly maximized in conjunction with weighted likelihoods of phrase contours. To find the optimal $\alpha$ value in Equation 6, we use the development data via a grid search. The optimal $\alpha$ is located at 0.25 . The corresponding RMSE and correlation coefficient between original and generated F0 contours are given in Table II. The RMSE and correlation coefficient are slightly improved comparing with generated by Equation 4. A high correlation coefficient of 0.92 achieved by only using DCT model on syllable level prevents it from being further improved significantly by integrating DCT model on phrase level. In addition, the dynamic features from proceeding and following syllables are jointly modeled and used to constrain tone contour generation in global sentence level. It can bring phrase-level intonation to a certain extent.
TABLE I. RMSE AND CORRELATION COEFFICIENTS BETWEEN ORIGINAL AND GENERATED F0 CONTOURS WITH STATIC AND DYNAMIC FEATURES

\begin{tabular}{|c|c|c|c|}
\hline Testing & Static & Static $+\Delta$ & Static $+\Delta+\Delta$ \\
\hline RMSE(Hz) & 20.59 & 19.81 & 19.54 \\
\hline Correlation & 0.911 & 0.919 & 0.921 \\
\hline
\end{tabular}

TABLE II. RMSE AND CORRELATION COEFFICIENTS BETWEEN ORIGINAL AND GENERATED F0 CONTOURS WITH/WITHOUT PHRASE-LEVEL DCT MODEL

\begin{tabular}{|l|c|c|}
\hline \multicolumn{1}{|c|}{ Testing } & $\boldsymbol{\alpha}=\mathbf{0}$ & $\boldsymbol{\alpha}=\mathbf{0 . 2 5}$ \\
\hline RMSE(Hz) & 19.54 & 19.45 \\
\hline Correlation & 0.921 & 0.922 \\
\hline
\end{tabular}

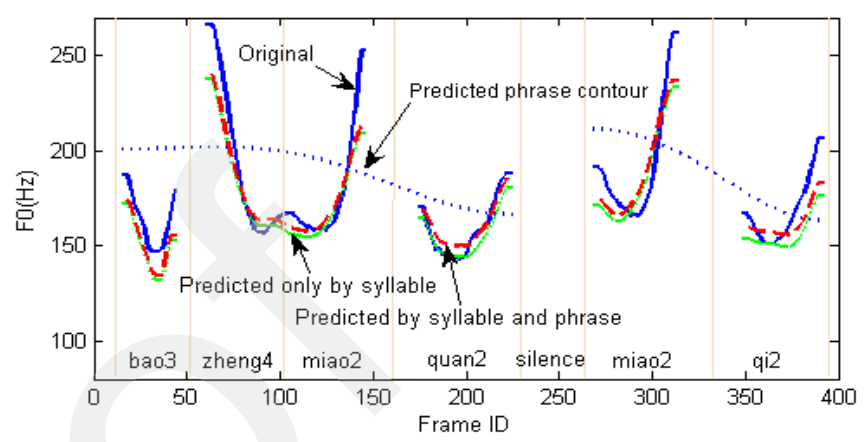

Figure 2. A sentence example of the original and generated F0 contours only by syllable and by syllable and phrase DCTs.

To analyze the generated phrase-level F0 contours, we cluster phrase-level DCT coefficients in terms of the number of syllables in phrase and the position of current phrase in sentence. The phrase-level F0 contours generated by these models are shown in Figure 3, where all contours descend from the beginning to the end of phrase with similar slopes, which seems independent of the number of constituent syllables. The majority of sentences in our corpus are declarative and uttered in broadcast news style. The observed phenomena are consistent with what mentioned in linguistics book. We also notice in Figure 3 that the starting and ending of phrase contours are correlated with the position of phrase in sentence.

\section{2) Speaker-independent isolated speech corpus:}

There are no tone contexts and phrases for the utterances in speaker-independent isolated speech corpus. The extracted F0s are normalized by the mean of the corresponding speaker. Tone-dependent DCT models are trained. The generated typical contours of four tones in Mandarin averaging DCT coefficients are shown in Figure 4. Table III shows the RMSE between original and generated F0 contours given certain speaker's F0 mean for testing data.

TABLE III. RMSE BETWEEN ORIGINAL AND GENERATED F0 TRAJECTORIES FOR SPEAKER INDEPENDENT CORPUS

\begin{tabular}{|l|c|}
\hline \multicolumn{1}{|c|}{ Testing } & Static \\
\hline RMSE(Hz) & 10.73 \\
\hline Correlation & 0.821 \\
\hline
\end{tabular}




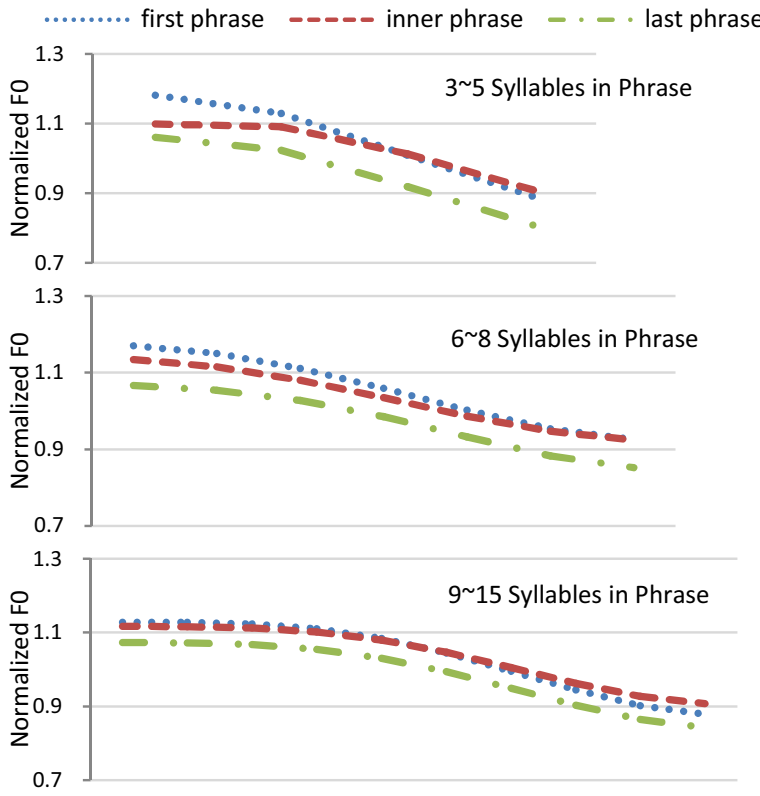

Figure 3. Generated phrase-level F0 contours

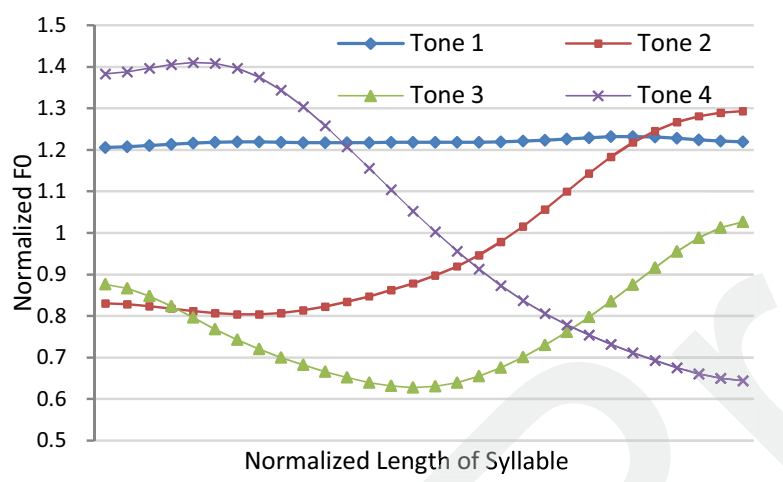

Figure 4. Typical contours of four tones in Mandarin averaging tonedependent DCT coefficients

\section{CONCLUSIONS}

DCT representation of F0 contours on syllable and phrase levels is used to model tone and intonation for Chinese Mandarin speech. The tone contours are generated by jointly optimizing the likelihoods of syllable and phrase DCT models in ML sense. The experimental results show our approach is positive and promising.

\section{REFERENCES}

[1] T. Yoshimura, K. Tokuda, T. Masuko, T. Kobayashi, and T. Kitamura, "Simultaneous Modeling of Spectrum, Pitch and Duration in HMM-based Speech Synthesis", Proc. of Eurospeech, 1999.

[2] A. W. Black, and A. J. Hunt, "Generating F0 Contours from ToBI Labels Using Linear Regression", Proc. of ICSLP, 1996.

[3] Q. Sun, K. Hirose and N. Minematsu, "Two-Step Generation of Mandarin F0 Contours Based on Tone Nucleus and Superpositional Models", Proc. of 6th ISCA Workshop on Speech Synthesis, 2007.

[4] Y. Qian, H. Liang and F. K. Soong, "Generating Natural F0 Trajectory with Additive Trees", Proc. of Interspeech 2008.

[5] X. Sun, "F0 Generation for Speech Synthesis Using a Multi-tier Approach", Proc. of ICSLP, 2002.

[6] S. Sakai, "Additive Modeling of English F0 Contour for Speech Synthesis", Proc. of ICASSP, 2005.

[7] J. Teutenberg, C. Watson and P. Riddle, "Modeling and Synthesizing F0 Contours with the Discrete Cosine Transform", Proc. of ICASSP, 2008.

[8] K. E. Dusterhoff, A. W. Black, and P. Taylor, "Using Decision Trees within the Tilt Intonation Model to Predict F0 Contours", Proc. of Eurospeech, 1999.

[9] K. Tokuda, T. Masuko, N. Miyazaki, and T. Kobayashi, "Multispace Probability Distribution HMM", IEICE Trans. Inf. \& Syst., E85-D(3), pp.455-464, 2002.

[10] Y. Chao, A Grammar of Spoken Chinese, Univ. of California Press, 1968.

[11] T. Hastie, R. Tibshirani, and J. Friedman, The Elements of Statistical Learning, Data Mining, Inference, and Prediction, Springer, 2001

[12] K. Shinoda, and T. Watanable, "MDL-based Context-dependent Sub-word Modeling for Speech Recognition", J. Acoust. Soc. $\operatorname{Jpn}(\mathrm{E})$, vol.21, no.2, pp.79-86, 2000.

[13] A.D. Talkin, "A Robust Algorithm for Pitch Tracking (RAPT)", In: Kleijn, W.B., Paliwal, K.K. (Eds.), Speech Coding and Synthesis, Elsevier Science BV, Amsterdam, pp. 495-518.

[14] Y. Qian, F. K. Soong, Y. Chen and M. Chu, "An HMM-based Mandarin Chinese Text-to-Speech System", Proc. of ISCSLP, 2006.

[15] K. Tokuda, T. Kobayashi, T. Masuko, T. Kobayashi and T. Kitamura, "Speech Parameter Generation Algorithms for HMMbased Speech Synthesis", Proc. of ICASSP, 2000. 